\title{
Commentary on "Two studies of Autonomous Sensory Meridian Response (ASMR): The Relationship between ASMR and Music-Induced Frisson" by Alexsandra Kovacevich and David Huron.
}

\author{
NICOLAS RUTH[1] \\ Media and Business Communication, University of Würzburg
}

Submitted 2018 October 26; accepted 2018 November 29.

KEYWORDS: ASMR, content analysis, frisson, YouTube

THE paper "Two studies of Autonomous Sensory Meridian Response (ASMR): The Relationship between ASMR and Music-Induced Frisson" is a reasonably done investigation of a new and viral phenomenon, called ASMR. The authors used two independent content analyses to identify the unique characteristics of ASMR videos and to investigate how people evaluate these videos.

I really enjoyed reading the paper and I have to admit that I now watched some of the videos. Afterward, I felt stunned and amused. ASMR is fascinating in an exceptional way, and I think the analyses done by Kovacevich and Huron help us to understand how these videos work and how recipients claim they react to them.

One of the most famous ASMR artists is called ASMR Darling. In 2018, she already has over 2 million followers on YouTube and several videos with over 10 and even 20 million views. In order to describe what ASMR is she uses the definition provided by the English Wikipedia: "Autonomous sensory meridian response (ASMR) is an experience characterized by a static-like or tingling sensation on the skin that typically begins on the scalp and moves down the back of the neck and upper spine. It has been compared with auditory-tactile synesthesia and may overlap with frisson." It seems that overlapping with frisson might be the right term as Kovacevich and Huron conclude, "ASMR might be reasonably regarded as a type of frisson."

\section{STUDY 1}

In study 1, the authors conduct a content analysis with which they compare ASMR videos to two control groups. The first consisted of a random selection of videos that fit the number of views and publishing date of the selected ASMR videos. The second group they called "talking-head control videos" featured tutorial videos that were comparable to ASMR in respect of camera focus on a talking protagonist and its head. The results reveal the seven most prominent themes of ASMR videos that are relaxation, quiet, proximity, intimacy, attraction, privacy, and movement. The comparison to the general control group seems a bit problematic and the authors appear to compare apples with a whole fruit salad. However, the comparison with the talking-head videos revealed the most relevant features of ASMP videos in terms of speech and audio.

\section{STUDY 2}

Study 2, in turn, focuses on the reactions that viewers claim to have experienced. To this end, the authors conducted a content analysis of user comments on ASMR videos. Especially comments that refer to physical and psychological reactions, the origins of those reactions, and the reasons for watching those videos were compiled for the analysis. Generally speaking, there is always an issue with identifying effects from content analyses. In the case of the current study, the authors gathered data from YouTube comments which means that the commentators are not aware that their responses were used for scientific analyses and are therefore not biased. However, at the same time, commentators might not mention everything they 
experienced in their comments. Similarly, commentators would hardly be able to articulate any subconscious effects they experienced while watching the videos. As the authors state, not all of the viewers leave comments. Furthermore, there might be a selection in the sense that those who leave comment might be ASMR fans and those who react to the videos in negative ways might not state their experiences. That is why these results should be validated in future, for example using survey data or physiological measurements.

\section{CONCLUSION}

Having said this, I still believe that the content analyses are an excellent starting point that provides insights that we might not get from survey data. Taken all together, the authors did an outstanding job in reviewing the ASMR literature and by discussing their results in comparison to previous empirical findings.

\section{ACKNOWLEDGEMENTS}

This article was layout edited by Kelly Jakubowski.

\section{NOTES}

[1] Correspondence can be addressed to: Dr Nicolas Ruth, Department of Media and Business Communication, University of Wuerzburg, Oswald-Külpe-Weg 82, 97074 Würzburg, Germany. E-mail: nicolas.ruth@uni-wuerzburg.de. 